\title{
Administración de estatinas durante la fase aguda del síndrome coronario agudo
}

\author{
M. Ruiz-Bailén \\ Unidad de Medicina Intensiva, Servicio de Cuidados Críticos y Urgencias, Departamento de Ciencias de la Salud, Complejo \\ Hospitalario de Jaén, Universidad de Jaén, Jaén, España
}

Recibido el 14 de julio de 2009; aceptado el 31 de julio de 2009

Disponible en Internet el 28 de octubre de 2009

\section{PALABRAS CLAVE \\ Mortalidad; \\ Infarto agudo de \\ miocardio; \\ Estatinas; \\ Síndrome coronario \\ agudo}

\section{KEYWORDS}

Mortality;

Acute myocardial

infarction;

Statins;

Acute coronary

syndrome

\begin{abstract}
Resumen
Es indiscutible el beneficio de las estatinas en los pacientes con síndrome coronario estable, pero en el síndrome coronario agudo (SCA) su administración precoz es controvertida. Existen pocos ensayos clínicos y metaanálisis sobre este tema, con resultados poco aclaratorios. Sin embargo, la mayoría de los registros la encuentra eficiente. No existe ningún estudio que encuentre inseguro este tratamiento, por lo que podría estudiarse su inclusión en las guías terapéuticas durante la fase aguda del SCA. (c) 2009 Elsevier España, S.L. y SEMICYUC. Todos los derechos reservados.
\end{abstract}

\begin{abstract}
Administration of statins during the acute phase of acute coronary syndrome
\section{Abstract}

The benefits of statins in patients with stable coronary syndrome is indisputable, however their early use in acute coronary syndrome (ACS) is under debate. There are few existing clinical trials and meta-analysis results on this and their results provide little clarification. However, most of the registries have found them to be efficient. There are no studies that show this therapy to be unsafe, so its inclusion in treatment guidelines during the acute phase of ACS could be studied.
\end{abstract}

(c) 2009 Elsevier España, S.L. and SEMICYUC. All rights reserved.
En la década de los cincuenta se impulsó el interés por controlar el colesterol. Durante esa época sólo se conocían las propiedades antilipidémicas de fármacos inseguros como el clofibrato, la niacina y la colestiramina. Por esto se comenzó la investigación sobre la producción hepática a

Correos electrónicos: ruizbailen@telefonica.net, mrb1604@terra.es.

URL: http://www.sccujaen.com través de la enzima hidroximetilglutaril-coenzima A reductasa ${ }^{1}$. En 1971, en Japón, el doctor Akira Endo descubrió las estatinas. El Dr. Akira aisló del Penicillium citrinum la molécula ML-236B, bautizada con posterioridad como mevastatina. Así, la mevastatina se convirtió en la primera molécula de la familia de las estatinas. Con posterioridad se aislaron y se sintetizaron nuevas estatinas ${ }^{1,2}$.

Múltiples y clásicos estudios han demostrado una marcada reducción del riesgo de episodios coronarios en pacientes 
estables y han dejado una indiscutible evidencia científica sobre la eficiencia de estos fármacos ante la enfermdedad coronaria estable $e^{3-9}$. Desgraciadamente no está aún claro ni definido el papel que presentan las estatinas en la fase aguda del síndrome coronario agudo (SCA), y menos aún su papel ante la fase aguda del infarto agudo de miocardio (IAM). De hecho, las actuales guías diagnóstico-terapéuticas europeas y americana de los distintos SCA e incluso sobre el tratamiento de los fármacos hipolipemiantes no dejan clara su indicación ${ }^{10-13}$. La acción de estas guías diagnósticoterapéuticas queda explicada por la falta de una evidencia absoluta de indicación en la fase aguda, dado los estudios existentes. Además de los estudios preclínicos existen muy pocos estudios que pueden confirmar el beneficio en la administración durante la fase aguda del IAM, e incluso crean una duda sobre dicho beneficio. En este apartado se va a realizar una revisión sobre los ensayos clínicos, los metaanálisis y los estudios observacionales existentes.

1. Ensayos clínicos (tabla 1): En el año 2001 se publicó el ensayo MIRACL (Myocardial Ischemia Reduction with Aggressive Cholesterol Lowering) ${ }^{14}$ realizado en Europa, América del Norte, Sudáfrica y Australasia. Los pacientes incluidos presentaron el diagnóstico de angina inestable (AI) o IAM no Q. El diagnóstico de Al requirió de apoyo diagnóstico objetivo. Los pacientes se excluyeron si: $a$ ) el colesterol total plasmático superaba los $270 \mathrm{mg} / \mathrm{dl}$; b) se planeó una revascularización coronaria previa; c) ante bloqueo de rama izquierda; d) ante ritmo de marcapasos; e) ante insuficiencia cardíaca congestiva grave; f) tratamiento concurrente con otros agentes que regulan los lípidos, o medicamentos asociados con rabdomiólisis; g) grave anemia; $h$ ) insuficiencia renal que requiere diálisis; i) disfunción hepática (definida por alaninaaminotransferasa superior a 2 veces el nivel superior normal); $j$ ) diabetes con dependencia a la insulina, y k) el embarazo o lactancia. Los pacientes se aleatorizaron, a doble ciego, entre las 24 y las $96 \mathrm{~h}$ después del ingreso en el hospital para recibir tratamiento con atorvastatina (80 mg/día) o placebo. Ambos grupos fueron homogéneos. La media de tiempo para recibir atorvastatina o placebo fue de $63 \mathrm{~h}$ en ambos grupos. Al inicio del estudio la media de los niveles séricos de colesterol unido a lipoproteínas de baja densidad (cLDL) fue de $124 \mathrm{mg} / \mathrm{dl}$, la del nivel de triglicéridos de $184 \mathrm{mg} /$ $\mathrm{dl}$ y la media de colesterol unido a lipoproteínas de alta densidad (cHDL) de $46 \mathrm{mg} / \mathrm{dl}$. Al final del estudio el cLDL había aumentado una media del $12 \%(135 \mathrm{mg} / \mathrm{dl})$ en el grupo placebo y había disminuido un $40 \%$ (72 mg/dl) en el grupo de la atorvastatina. Los triglicéridos aumentaron a $187 \mathrm{mg} / \mathrm{dl}$ en el grupo placebo y disminuyeron una media del $16 \%(139 \mathrm{mg} / \mathrm{dl})$ en el grupo de la atorvastatina. Los cambios en el cHDL durante el estudio fueron menores en ambos grupos. Durante las 16 semanas del seguimiento el objetivo principal primario se produjo en 228 pacientes $(14,8 \%)$ del grupo de la atorvastatina y en 269 pacientes $(17,4 \%)$ del grupo placebo, con una diferencia absoluta del $2,6 \%$ y con una diferencia relativa del $16 \%$; traducido en un número necesario para tratar (NNT) de 38 . El tratamiento con atorvastatina redujo significativamente el riesgo combinado del objetivo final primario (riesgo relativo [RR]: 0,84; intervalo de confianza [IC] del 95\%: $0,70-1,00 ; p=0,048)$. No se observaron diferencias en el riesgo de muerte, IAM no mortales, o paro cardíaco con reanimación entre ambos grupos; aunque el grupo de atorvastatina tuvo un menor riesgo de síntomas recurrentes de isquemia miocárdica, evaluados con pruebas objetivas que requirieron hospitalización de emergencia (RR: 0,74; IC del 95\%: 0,57-0,95; $p=0,02$ ). No hubo interacción entre ninguna variable, y en especial con los niveles séricos de lípidos. El accidente cerebrovascular (ACV) no mortal se produjo en 9 pacientes en el grupo de atorvastatina frente a 22 pacientes en el grupo placebo (RR: 0,41; IC del 95\%: $0,20-0,87 ; p=0,02 ;$ NNT de 33). La elevación anormal de las aminotransferasas (definida como aquella que presenta elevación en más de 3 veces el doble del valor normal) se produjo en 38 pacientes (2,5\%) en el grupo de atorvastatina y en 9 pacientes $(0,6 \%)$ en el grupo placebo $(p=0,001 ;$ NNT de 53). En resumen, este ensayo clínico encuentra que la administración de atorvastatina en las primeras 24-96 h en pacientes con SCA: a) disminuye los episodios de isquemia recurrente; b) disminuye la variable combinada de efectos cardiovasculares y c) disminuye la incidencia de ACV isquémicos. Estos efectos fueron independientes del nivel de lípidos. No obstante, falló en encontrar una disminución de la mortalidad. Se establece así la necesidad de iniciar el tratamiento con estatinas precozmente, antes del alta hospitalaria, aunque no indica una administración precoz en el momento inicial del SCA (tabla 1).

El estudio "A to Z" ${ }^{15}$ incluyó a los supervivientes de la fase A de dicho ensayo, donde se comparaba la enoxaparina frente a la heparina no fraccionada. Se incluyó en el estudio a los pacientes estables con IAM y con Al. Se administró de forma aleatorizada simvastatina en dosis de $40 \mathrm{mg}$ durante un mes seguido de $80 \mathrm{mg}(\mathrm{n}=2.265)$ frente a placebo durante 4 meses seguido de $20 \mathrm{mg}$ de simvastatina $(n=2.232)$. En el $44 \%$ de los pacientes se realizó intervencionismo coronario percutáneo (ICP) y se administró antillb/llla en el 50\%. En el grupo placebo se incrementó el cLDL un $11 \%$ a los 4 meses y descendió a los 8 meses hasta $77 \mathrm{mg} / \mathrm{dl}$ (20 mg de simvastatina) y en el grupo de simvastatina descendió inicialmente hasta $68 \mathrm{mg} / \mathrm{dl}$ (39\%) en el primer mes y hasta $62 \mathrm{mg} / \mathrm{dl}$ a los 4 meses. El objetivo primario ocurrió en el $16,7 \%$ en el grupo placebo frente al $14,4 \%$ para la simvastatina (RR: 0,89; IC del 95\%: 0,79-1,04). Se produjo miopatía (definida por la presencia de síntomas musculares y elevación de la creatincinasa unas 10 veces el valor del límite superior) en 9 pacientes $(0,4 \%)$ del grupo de simvastatina $(80 \mathrm{mg} / \mathrm{dl})$ y en un paciente del grupo placebo $(p=0,02)$. Se abandonó el tratamiento por problemas musculares en el $1,5 \%$. Los mismos autores señalan que el resultado "fallido" podría explicarse por el retraso en la administración de estatinas y porque le falta potencia al estudio al abandonar el tratamiento más de un 33\% de los pacientes.

Este ensayo clínico no fue capaz de demostrar que el inicio precoz e intensivo de estatinas tras un SCA resulte superior a un régimen de inicio posterior y menos intensivo; pues aunque presentó una tendencia favorable de reducción de los episodios cardiovasculares mayores, este beneficio no llegó a tener significación estadística (tabla 1). 
Tabla 1 Resumen de los distintos ensayos clínicos que evalúan las estatinas durante la fase precoz del síndrome coronario agudo (SCA)

\begin{tabular}{|c|c|c|c|c|c|c|c|c|c|c|c|}
\hline Ensayo $^{a}$ & $\mathrm{n}$ & Selección & Intervención & $\begin{array}{l}\text { Objetivo } \\
\text { primario }\end{array}$ & $\begin{array}{l}\text { Objetivos } \\
\text { secundarios }\end{array}$ & Seguimiento & $\begin{array}{l}\text { Momento de } \\
\text { aleatorización }\end{array}$ & Resultados & Abandono & $\begin{array}{l}\text { Elevación de } \\
\text { aminotransferasa }\end{array}$ & Miopatías \\
\hline MIRACL & 3.086 & SCA & $\begin{array}{l}80 \mathrm{mg} \text { de } \\
\text { atorvastatina frente } \\
\text { a placebo }\end{array}$ & $\begin{array}{l}\text { Muerte, } \\
\text { nuevo IAM, } \\
\text { parada } \\
\text { cardíaca o } \\
\text { isquemia } \\
\text { miocárdica }\end{array}$ & $\begin{array}{l}\text { Cualquier variable } \\
\text { individual del } \\
\text { objetivo primario, } \\
\text { ACV, insuficiencia } \\
\text { cardíaca o } \\
\text { necesidad de } \\
\text { revascularización y } \\
\text { niveles séricos de } \\
\text { lípidos }\end{array}$ & 16 semanas & $\begin{array}{l}\text { 24-96h } \\
\text { (media 63 h) }\end{array}$ & $\begin{array}{l}\text { El objetivo } \\
\text { primario tuvo } \\
\text { RR: } 0,84 \text {; IC del } \\
95 \%: 0,70-1,00 ; \\
\mathrm{p}=0,48 \text {. } \\
\text { Disminución de } \\
\text { isquemia } \\
\text { recurrente y de } \\
\text { ACV }\end{array}$ & $\begin{array}{l}\text { El } 14 \text { para el } \\
\text { grupo de la } \\
\text { atorvastatina } \\
\text { frente al } 12 \% \\
\text { para el grupo } \\
\text { del placebo }\end{array}$ & $\begin{array}{l}\text { El } 2,5 \text { para la } \\
\text { atorvastatina } \\
\text { versus el } 0,6 \% \\
\text { para el placebo }\end{array}$ & 0 \\
\hline$A$ to $Z$ & 4.497 & SCA & $\begin{array}{l}\text { Simvastatina de } \\
40 \text { mg durante un } \\
\text { mes, seguido por } \\
80 \text { mg de } \\
\text { simvastatina, versus } \\
\text { placebo durante } 4 \\
\text { meses, seguido por } \\
20 \text { mg de } \\
\text { simvastatina }\end{array}$ & $\begin{array}{l}\text { Muerte o } \\
\text { nuevo IAM, } \\
\text { ACV } \\
\text { isquémico } \\
\text { o isquemia } \\
\text { miocárdica }\end{array}$ & $\begin{array}{l}\text { Cualquier variable } \\
\text { individual del } \\
\text { objetivo primario }\end{array}$ & 4-24 meses & $\begin{array}{l}\text { Media de 3,7 } \\
\text { días }\end{array}$ & $\begin{array}{l}\text { A los } 4 \text { meses } \\
\text { siguientes el } \\
\text { objetivo } \\
\text { principal tuvo } \\
\text { RR: } 0,75 \\
(0,60-0,95)\end{array}$ & $\begin{array}{l}\text { El } 34 \text { versus el } \\
32 \% \text { para el } \\
\text { placebo }\end{array}$ & $\begin{array}{l}\text { El } 0,9 \text { versus el } \\
0,4 \% \text { para el } \\
\text { placebo }\end{array}$ & $\begin{array}{l}\text { El 0,4 versus el } \\
0,04 \% \text { para el } \\
\text { placebo }\end{array}$ \\
\hline $\begin{array}{l}\text { PROVE } \\
\text { IT-TIMI } \\
22^{\mathrm{b}}\end{array}$ & 4.162 & $\mathrm{SCA}$ & $\begin{array}{l}\text { Atorvastatina de } \\
80 \mathrm{mg} \text { frente a } \\
40 \mathrm{mg} \text { de } \\
\text { pravastatina }\end{array}$ & $\begin{array}{l}\text { Muerte o } \\
\text { nuevo IAM, } \\
\text { ACV } \\
\text { isquémico } \\
\text { o isquemia } \\
\text { miocárdica }\end{array}$ & $\begin{array}{l}\text { Cualquier variable } \\
\text { individual del } \\
\text { objetivo primario }\end{array}$ & $\begin{array}{l}24 \text { meses } \\
\text { (media } \\
18-36)\end{array}$ & $\begin{array}{l}\text { Los primeros } \\
10 \text { días }\end{array}$ & $\begin{array}{l}\text { Superioridad } \\
\text { del régimen de } \\
80 \mathrm{mg} \text { de la } \\
\text { atorvastatina }\end{array}$ & $\begin{array}{l}\text { El 21,4 para } \\
\text { la } \\
\text { atorvastatina } \\
\text { versus el } \\
22,8 \% \text { para la } \\
\text { pravastatina }\end{array}$ & $\begin{array}{l}\text { El } 1,1 \text { para la } \\
\text { pravastatina } \\
\text { frente al } 3,3 \% \\
\text { para la } \\
\text { atorvastatina }\end{array}$ & $\begin{array}{l}\text { El } 2,7 \text { para la } \\
\text { pravastatina } \\
\text { versus el 3,3\% } \\
\text { para la } \\
\text { atorvastatina }\end{array}$ \\
\hline FLORIDA & 540 & IAM & $\begin{array}{l}80 \mathrm{mg} \text { de } \\
\text { fluvastatina frente a } \\
\text { placebo }\end{array}$ & $\begin{array}{l}\text { Muerte o } \\
\text { nuevo IAM } \\
\text { o isquemia } \\
\text { miocárdica }\end{array}$ & $\begin{array}{l}\text { Cualquier variable } \\
\text { individual del } \\
\text { objetivo primario }\end{array}$ & 12 meses & 8 días & $\begin{array}{l}\text { No hay } \\
\text { diferencias }\end{array}$ & $\begin{array}{l}\text { El } 11,3 \text { para } \\
\text { fluvastatina } \\
\text { versus el } \\
13,5 \% \text { para } \\
\text { placebo }\end{array}$ & - & - \\
\hline PACT & 3.408 & SCA & $\begin{array}{l}\text { Pravastatina ( } 20 \text { o } \\
40 \mathrm{mg} \text { ) versus } \\
\text { placebo }\end{array}$ & $\begin{array}{l}\text { Muerte o } \\
\text { nuevo IAM } \\
\text { o isquemia } \\
\text { miocárdica }\end{array}$ & $\begin{array}{l}\text { Cualquier variable } \\
\text { individual del } \\
\text { objetivo primario }\end{array}$ & 4 semanas & $24 \mathrm{~h}$ & $\begin{array}{l}\text { No hay } \\
\text { diferencias }\end{array}$ & - & $\begin{array}{l}\text { El } 1,5 \text { para la } \\
\text { pravastatina } \\
\text { versus el } 1,1 \% \\
\text { para el placebo }\end{array}$ & 0 \\
\hline
\end{tabular}


El ensayo PROVE IT-TIMI $22^{16}$ (Pravastatin or Atorvastatin Evaluation and Infection Therapy-Thrombolysis in Myocardial Infarction 22 ) reclutó a 4.162 pacientes con SCA diagnosticado en los últimos 10 días, salvo inestabilidad. Además, los pacientes tenían que tener un nivel de colesterol total superior o igual a $240 \mathrm{mg} / \mathrm{dl}$; se excluyeron cuando habían recibido fármacos inhibidores del citocromo P-450 en el último mes, o les habían realizado ICP en los últimos 6 meses o un by-pass en los últimos 2 meses, ante factores que alarguen el intervalo QT corregido, o enfermedad hepática. El seguimiento medio fue de 24 meses (18-36). Los pacientes se aleatorizaron para recibir $80 \mathrm{mg}$ de atorvastatina $040 \mathrm{mg}$ de pravastatina. Los 2 grupos fueron homogéneos, salvo para una mayor historia de enfermedad vascular en el grupo de la pravastatina. La edad media fue de 58 años y el $22 \%$ eran mujeres. Al inicio la media de los niveles de cLDL fue de $106 \mathrm{mg} / \mathrm{dl}$. Al mes el cLDL logrado durante el seguimiento fue de $95 \mathrm{mg} / \mathrm{dl}$ $(79-113 \mathrm{mg} / \mathrm{dl}$ ) en el grupo de pravastatina y de $62 \mathrm{mg} / \mathrm{dl}$ $(50-79 \mathrm{mg} / \mathrm{dl})$ en el grupo de atorvastatina $(p<0,001)$. Entre los 990 pacientes (25\%) que habían recibido previamente estatinas, el cLDL permaneció sin cambios en los niveles basales en el grupo de pravastatina, mientras que se redujo un $32 \%$ en el grupo de atorvastatina $(p<0,001)$. La media de la proteína $C$ reactiva disminuyó los niveles desde 12,3 a 2,1 mg/l en el grupo de la pravastatina y a $1,3 \mathrm{mg} / \mathrm{l}$ en el grupo de la atorvastatina $(p<0,001)$. A los 30 días la frecuencia observada para la pravastatina del objetivo primario fue del 26,3 y del $22,4 \%$ para la atorvastatina, lo que representa una reducción del $16 \%$ del riesgo a favor de la atorvastatina ( $p=0,005$; IC del 95\%: 5-26\%). El riesgo de muerte, IAM, o revascularización urgente se redujo un $25 \%$ en el grupo de la atorvastatina $(p<0,001)$. Entre los componentes individuales del objetivo primario hubo un beneficio a favor de las altas dosis de atorvastatina con un $14 \%$ de reducción en la necesidad de revascularización $(p=0,04)$ y un $29 \%$ de reducción en el riesgo de $\mathrm{Al}$ recurrentes $(p=0,02)$. El ACV fue infrecuente y las tasas no difirieron significativamente entre los grupos. Este ensayo demostró, en los pacientes que habían presentado recientemente un SCA, la superioridad de un régimen hipolipemiante con estatinas más intensivo en la protección frente a la muerte y episodios cardiovasculares mayores; y no sólo a largo plazo como se había encontrado en estudios previos, sino también de forma muy precoz, en el primer mes (tabla 1).

El objetivo del ensayo del Fluvastatin on Risk Diminishment After Acute Myocardial Infarction (FLORIDA) Study Group $^{17}$ fue evaluar la disminución de isquemia tras el IAM mediante la administración de $40 \mathrm{mg}$ de fluvastatina. Diseñaron un ensayo clínico aleatorizado, a doble ciego, cuyo objetivo primario fue la variable combinada de muerte o isquemia recurrente que requiera hospitalización o revascularización. El seguimiento fue de 12 meses. Tras 12 meses la fluvastatina disminuyó el cLDL un $21 \%$ (de 134 a $103 \mathrm{mg} / \mathrm{dl} ; \mathrm{p}<0,0001$ ) comparado con un incremento del $9 \%$ en el grupo placebo. No encontraron ninguna diferencia significativa entre la fluvastatina y el placebo en términos de mortalidad o de reducción de isquemia miocárdica (el $2,6 \%$ en el grupo de la fluvastatina frente al $4 \%$ en el control). El ensayo FLORIDA resultó poco potente para aceptar o descartar un efecto de la fluvastatina en la isquemia miocárdica tras el IAM, medida mediante electrocardiografía ambulatoria. Dichos resultados podrían explicarse por la baja muestra incluida $y$, quizás, por la dosis del fármaco (tabla 1).

Thompson et al ${ }^{18}$ evaluaron la eficacia y seguridad del uso de la pravastatina utilizada en las primeras $24 \mathrm{~h}$ del inicio de cualquier tipo de SCA. Se excluyeron aquellos pacientes con tratamiento previo con estatinas. Se aleatorizaron mediante doble ciego a recibir 20 o $40 \mathrm{mg} / 24 \mathrm{~h}$ de pravastatina frente a placebo (al principio del estudio comenzaron con $20 \mathrm{mg}$ [ $\mathrm{n}=720]$ y tras comprobar su seguridad se administraron $40 \mathrm{mg}[\mathrm{n}=990])$. El seguimiento fue de 30 días. El cálculo de tamaño muestral requerido fue de 10.000 pacientes. No obstante, sólo se incluyó a 2.006 pacientes con IAM y 1.402 pacientes con Al. Se excluyó del estudio a 441 pacientes ya que no se confirmó el diagnóstico de SCA. Dicho objetivo se produjo en el 11,6\% del grupo de la pravastatina frente al $12,4 \%$ del grupo de placebo (no significativo). Sin embargo, este fármaco sí se mostró seguro al no detectarse incrementos de complicaciones asociadas. El ensayo PACT (Pravastatin in Acute Coronary Treatment) encuentra un efecto favorable, aunque no significativo, en el pronóstico a los 30 días de la pravastatina administrada en las $24 \mathrm{~h}$ del SCA, y resulta segura su administración. Los autores del PACT no logran encontrar beneficio en la administración precoz de estatinas, aunque no llegan a reclutar la muestra requerida y además utilizan una dosis de estatinas variablemente baja (tabla 1).

2. Metaanálisis: Cannon et $\mathrm{al}^{19}$ incluyeron en su metaanálisis 27.548 pacientes con SCA de 4 ensayos clínicos. Hubo un claro descenso significativo de los niveles de CLDL en los tratados con altas dosis de estatinas (el 22 frente al $42 \%$; media de $75 \mathrm{mg} / \mathrm{dl} ; \mathrm{p}<0,001$; lo que supone una disminución del $25,7 \%$ ). El análisis mostró una reducción del $16 \%$ de la muerte cardiovascular o el IAM (el 9,4 frente al 8,0\%; odds ratio [OR]: 0,84; IC del 95\%: 0,77-0,91; $\mathrm{p}<0,00001$ ). También redujo un $16 \%$ la muerte coronaria o cualquier episodio cardiovascular (el 32,3 frente al 28,8\%; OR 0,84; IC del 95\%: 0,8-0,89; $\mathrm{p}<0,00001$ ). Extrapolando los datos de este metaanálisis se podría indicar que por cada millón de pacientes tratados por SCA durante 5 años con el tratamiento intensivo se podrían prevenir 35.000 episodios cardiovasculares (incluyendo 14.000 muertes coronarias o por IAM). El NNT de este trabajo es de 29. No obstante, este metaanálisis se realizó sobre una población estable y con SCA, se desconoce el momento de inicio de los síntomas y, además, se desconoce el tiempo de seguimiento. Hulten et $\mathrm{al}^{20}$ realizaron un metaanálisis en el que incluyeron 17.963 pacientes de 13 ensayos clínicos. Su selección se basó en la administración de estatinas en los primeros 14 días tras el ingreso por un SCA. La mayoría de los ensayos tenían unas muestras inferiores a 100 pacientes y utilizaban objetivos angiográficos y otros para prevención secundaria. Definieron la administración de estatinas en dosis altas como al menos el doble de la habitual. La administración se inició entre 1 y 14 días, media de 4 días. No encontraron diferencias en cuanto a las diferencias cardiovasculares en los 4 primeros meses, 
aunque sí en los primeros 6 meses (RR: 0,76; IC del 95\%: $0,70-0,84)$; un beneficio que persistió tras 24 meses de seguimiento (RR: 0,81 ; IC de 95\%: 0,77-0,87). A los 24 meses de seguimiento se observó una disminución de la mortalidad (RR: 0,76; IC del 95\%: 0,66-0,87) e isquemia miocárdica (RR: 0,68; IC del 95\%: 0,50-0,92), pero no de IAM (RR: 0,89; IC del 95\%: 0,60-1,33). El grupo tratado con estatinas redujo la media de cLDL en $34 \pm 9 \mathrm{mg} / \mathrm{dl}$ $(p<0,001)$. Sólo encontraron 3 casos de rabdomiólisis (simvastatina).

Briel et $\mathrm{al}^{21}$ evalúan la administración de las estatinas, iniciada en los 14 primeros días del SCA y seguidas tras 2 a 4 meses. El objetivo primario fue la variable combinada de IAM no fatal, ACV no fatal, muerte cardiovascular y muerte global. Los objetivos secundarios fueron los desagregados del objetivo primario más el procedimiento de revascularización o isquemia miocárdica que requirió reingreso. En 12 ensayos clínicos incluidos en el metaanálisis, con un total de 13.024 pacientes con SCA, se evaluaron 4 estatinas diferentes (pravastatina de 6 ensayos: 3 con atorvastatina, 2 con fluvastatina y uno con simvastatina). Muchos ensayos eran para prevención secundaria y otros con estudios meramente angiográficos. La media de cLDL al ingreso fue de $123 \mathrm{mg} / \mathrm{dl}$ (112-178), la reducción media fue del 15 al 53\% y la media en el descenso del colesterol total fue del 9 al $37 \%$. La reducción fue mayor con las dosis más altas de colesterol. La acción sobre el CHDL y los triglicéridos fue menos intensa. Durante el primer mes de seguimiento el $4,7 \%$ de los tratados con estatinas fallecieron o presentaron IAM o ACV. En el grupo control este episodio ocurrió en el 5\% (RR: 0,93; IC del 95\%: 0,8-1,09). A los 4 meses de seguimiento presentaron el episodio combinado el 7,5 frente al 8,1\% en el grupo control (RR: 0,93; IC de 95\%: 0,81-1,07). Al mes tampoco hubo diferencias entre los objetivos secundarios. A los 4 meses la Al se redujo con las estatinas (el 4,8 frente al 6\%; RR: 0,8; IC del 95\%: $0,64-1 ; p=0,05)$. La heterogeneidad entre los grupos fue mínima, salvo en el caso de la Al.

3. Registros: Stenestrand et $\mathrm{al}^{22}$ evaluaron mediante un registro clínico, realizado exclusivamente con pacientes con IAM y edad menor de 80 años, la mortalidad al año entre los pacientes tratados con estatinas y sin estatinas antes del alta hospitalaria. Durante 1995-1998 se incluyeron en el estudio 14.071 pacientes sin estatinas y 5.528 pacientes con estatinas. La mortalidad no ajustada fue del 9,3 frente al $4 \%$ en el grupo de estatinas. El análisis de propensión mostró una reducción de la mortalidad al año (OR: 0,75; IC del 95\%: 0,63-0,89). El beneficio en la disminución de la mortalidad de las estatinas también se detectó mediante la regresión de Cox (RR: 0,5; IC del 95\%: 0,36-0,69).

De los pacientes de los ensayos clínicos GUSTO IIb y del PURSUIT se evaluó el efecto de la administración de las estatinas sobre la mortalidad global ${ }^{23}$. Recibieron estatinas 3.653 pacientes frente a 17.156 pacientes. La edad mediana de estos 20.809 pacientes fue de 64 años. En el grupo tratado con estatinas hubo más mujeres, fueron más jóvenes, más obesos, más hipertensos, más hiperlipidémicos, más diabetes mellitus, más enfermedad vascular periférica, más IAM y revascularización previa. Al mismo tiempo este grupo se trató de forma más habitual con aspirina, inhibidores de la enzima de conversión de angiotensina (IECA) y bloqueadores beta. A los 6 meses de seguimiento de los pacientes tratados con estatinas, las curvas de Kaplan-Meier mostraron una mejoría de la supervivencia. A los 30 días del seguimiento persistió la reducción por cualquier causa de mortalidad (el 1,7 frente al 3,5\%; OR: 0,48; IC del 95\%: 0,37-0,63). A los 6 meses el modelo multivariado ajustado al análisis de propensión mostró que las estatinas se comportan como variable independiente frente a la variable final de muerte o infarto no fatal (OR: 0,8; IC del 95\%: 0,65-0,98; $\mathrm{p}=0,035)$. Las estatinas presentaron una disminución absoluta de la mortalidad del 1,8\% y un NNT de 56 a los 6 meses.

Newby et $\mathrm{al}^{24}$ realizaron un estudio observacional (SYMPHONY y SYMPHONY 2) e incluían a 12.365 pacientes con SCA. Aunque entre los primeros 90 días y el año resultó una menor mortalidad no ajustada para los 90 días (RR: 0,58; IC del 95\%: 0,42-0,81) y para la mortalidad al año (RR: 0,52; IC del 95\%: 0,40-0,68). Sin embargo, este beneficio no fue evidente durante los primeros 90 días. Además, tras realizar el análisis de propensión no hubo diferencias entre la administración precoz ni la tardía para el resultado de muerte, IAM o isquemia grave recurrente. Por tanto, los datos de este registro indican que la administración precoz de estatinas no genera ningún beneficio.

De los 3.232 pacientes del "Platelet Receptor Inhibition in Ischemic Syndrome Management" ${ }^{25}$, se investigó a 1.616 pacientes con SCA (1.249 sin estatinas, 379 con tratamiento continuado con estatinas y 86 con tratamiento con estatinas que se suspendió tras la hospitalización). El objetivo primario fue la variable combinada de muerte, IAM o angina recurrente en las últimas $48 \mathrm{~h}$. El objetivo secundario fue la muerte, IAM 0 isquemia recurrente a los 7 y a los 30 días de seguimiento. Ambos grupos fueron homogéneos. La administración de estatinas se asoció con una reducción de episodios a los 30 días de seguimiento, comparado con los pacientes sin estatinas (OR: 0,49; IC del 95\%: 0,21-0,86). La isquemia recurrente fue significativamente menor en el grupo de las estatinas a las $48 \mathrm{~h}$ del seguimiento (OR: 0,35; IC del 95\%: $0,16-0,89)$, aunque no a los 30 días. La revascularización también fue significativamente menor en los pacientes tratados con estatinas. La estancia media fue menor en los pacientes con estatinas $(11,5$ frente a 9,2 días; $p=0,002$ ). En los pacientes en los que se retiraron las estatinas tras el ingreso aumentó el riesgo de episodios cardiovasculares (OR: 2,93; IC del 95\%: $1,64-6,27)$, que se incrementaron directamente tras la primera semana de la retirada de las estatinas, con independencia del nivel de colesterol. En la regresión logística las variables asociadas al pronóstico del paciente fueron la troponina, los cambios del segmento ST y la discontinuación con las estatinas. La mayoría de los pacientes del grupo de estatinas recibieron simvastatina (50\%), seguido por lovastatina $(24,1 \%)$ y pravastatina $(20,4 \%)$. En el grupo de la simvastatina, el $85,2 \%$ de los pacientes recibió entre 10 y $20 \mathrm{mg}$, y menos del $10 \%$ se trató con altas dosis de estatinas. Un CLDL superior a $200 \mathrm{mg} / \mathrm{dl}$ se observó en el $43 \%$ del grupo de estatinas y en el $58 \%$ de los pacientes que no recibieron estatinas. 
Entre 1999 y 2002 se incluyeron 19.537 pacientes con SCA en el estudio GRACE (Gender, Race, and Clinical Experience $)^{26}$. El tratamiento se definió sobre la base de la administración de estatinas en los últimos 7 días. $\mathrm{El}$ grupo de administración precoz se definió de acuerdo al inicio del tratamiento en las primeras $24 \mathrm{~h}$. Entre éstos, 4.056 pacientes $(21 \%)$ estaban tomando estatinas previamente, de los que $428(11 \%)$ no las recibieron durante la fase aguda de hospitalización. Los pacientes que tomaban estatinas previamente presentaron con menor frecuencia elevación del segmento ST (OR: 0,75; IC del 95\%: $0,71-0,88)$. No encontraron interacción entre la hiperlipidemia y las estatinas. Los predictores de discontinuación del tratamiento con estatinas fueron: la edad, la administración de aspirina, el sexo masculino, y fumar tabaco. Los pacientes que mantuvieron el tratamiento con estatinas recibieron con más frecuencia otros fármacos "cardioprotectores" como aspirina u otros antitrombóticos (enoxaparina, IECA, bloqueadores beta o inhibidores de la glucoproteina Ilb/IIla). Los pacientes que recibieron previamente estatinas y que continuaron el tratamiento con éstas tuvieron menos probabilidades de morir en el hospital (OR: 0,38; IC del 95\%: 0,28 a 0,52) que los pacientes que nunca recibieron estatinas. Sin embargo, en los pacientes que previamente tomaban estatinas, pero que se discontinuaron en el episodio actual, no hubo diferencias significativas en la mortalidad (OR: 1,39; IC del 95\%: 0,91-2,14). De los 15.481 pacientes que previamente no tomaban estatinas, 5.959 pacientes (38\%) las recibieron en el hospital. Estos pacientes eran más jóvenes, más frecuentemente varones, con menor comorbilidad, más fumadores e hiperlipidémicos que los pacientes que nunca recibieron estatinas. También tomaban antes del ingreso con menos probabilidad aspirina, bloqueadores beta o IECA. Además, estos pacientes estaban menos críticos, con menos posibilidad de taquicardia sinusal o hipotensión, ausencia de clase Killip avanzado; pero eran más proclives a presentar elevaciones del segmento ST. Estos pacientes, además, fueron más propensos a los tratamientos con otros fármacos cardioefectivos o ICP que los pacientes que nunca recibieron estatinas. Los pacientes que iniciaron estatinas durante la hospitalización tuvieron menor mortalidad (OR: 0,38; IC del 95\%: $0,3-0,48)$.

Foranow et $\mathrm{al}^{27}$ mediante el registro NRMI 4, incluyeron 174.635 pacientes con IAM y evaluaron la administración de estatinas. La administración nueva o la continuación del tratamiento con estatinas en las primeras $24 \mathrm{~h}$ se asoció con un descenso de la mortalidad en comparación con el no uso de las estatinas (el 4,0 y el 5,3 vs. el $15,4 \%$ en los que no usaron estatinas). La administración precoz de estatinas se asoció a una menor incidencia de choque cardiogénico, arritmias, parada cardíaca y rotura cardíaca, pero no de IAM recurrente. El análisis multivariante mostró una disminución de la mortalidad para los grupos de tratamiento con estatinas (OR de 0,46 para la continuación del tratamiento, OR de 0,42 para el inicio de un nuevo tratamiento y OR de 1,25 para la discontinuación del tratamiento). Tras ajustar por las posibles variables confusoras y el análisis de propensión para las estatinas, los pacientes que continuaron con estatinas tuvieron una disminución de la mortalidad (OR: 0,62; IC del 95\%: 0,57-0,67) en comparación con los pacientes no tratados.

El Euro Heart Survey ${ }^{28}$ incluyó pacientes con SCA y evaluó el efecto de la administración de estatinas en las primeras $24 \mathrm{~h}$ del ingreso. Se realizó un seguimiento de 7 días en 7.696 pacientes (94\%) y de 30 días en 6.899 pacientes $(84 \%)$. La mortalidad global a los 7 días fue significativamente menor en el grupo de los pacientes que recibieron el tratamiento con estatinas (el 0,4 frente al 2,6\% de episodios; el 2,2\% de diferencia absoluta; RR ajustado: 0,34; IC del 95\%: 0,15-0,79). Las diferencias estadísticamente significativas se observaron en los pacientes con SCA con elevación del segmento ST (RR ajustado: 0,17; IC del 95\%: 0,04-0,70), pero no en los SCA sin elevación del segmento ST.

\section{Discusión}

La enfermedad ateroesclerótica es actualmente la causa mundial de mayor morbimortalidad. Si bien es cierto que la revascularización precoz del SCA ha logrado una notable mejoría del pronóstico, esta medida es insuficiente, se requiere además una estrategia preventiva y terapéutica farmacológica. En esta dirección es indiscutible el beneficio atribuible de las estatinas, se estima que desde su descubrimiento han podido salvar más de 3 millones de vidas humanas. Este beneficio se cree atribuido a la prevención de episodios cardiovasculares y se ha demostrado claramente en los pacientes con enfermdedad cardiovascular estable. Sin embargo, en la actualidad se mantiene una clara controversia sobre su utilidad en la fase aguda del SCA. Existen muchos estudios experimentales que nos han hecho comprender las distintas vías de actuación de estos fármacos y sus posibles efectos beneficiosos, que expanden el concepto del beneficio de ser un meramente hipolipemiante a poseer efectos antiinflamatorios y antiproliferativos descritos como “efectos pleiotrópicos". Existen estudios experimentales humanos, amplios en número, pero con bajo nivel de evidencia y dirigidos fundamentalmente hacia objetivos combinados $u$ objetivos fundamentalmente angiográficos, que han encontrado en la mayoría de éstos ciertos beneficios; no obstante, son estudios muy heterogéneos, por lo que es difícil extraer conclusiones evidentes. Desgraciadamente, existen pocos ensayos clínicos dirigidos hacia la evaluación de las estatinas en la fase aguda del IAM. Estos estudios con mayor nivel de evidencia (ensayos clínicos en fase III) nos siguen manteniendo una duda razonable sobre la eficacia de las estatinas. Dichos ensayos utilizan una metodología muy variada, con poblaciones muy heterogéneas (IAM y Al), con objetivos diferentes (nivel de lípidos, inflamación, isquemia miocárdica, morbimortalidad, etc.), y requieren en muchas ocasiones variables clínicas combinadas (suelen evaluar objetivos primarios distintos, pero todos éstos requieren variables combinadas para obtener resultados, mezclan a menudo dichos objetivos, y realizan evaluaciones a posteriori e incluso mezclan objetivos finales). E incluso en muchos de dichos ensayos clínicos se considera criterio de exclusión al "paciente 
inestable", situación que suele ser habitual ante un SCA; por lo tanto, no se puede descartar en tales ensayos clínicos un "probable sesgo de selección”. Además, utilizan fármacos variados y en dosis variadas, e inician la aleatorización en momentos distintos. Algunos de los ensayos clínicos podrían explicar la falta de eficacia por no alcanzar la potencia requerida, otros por la tardanza en administrar las estatinas. En definitiva, los ensayos clínicos existentes muestran múltiples déficits que aunque nos hacen difícil evaluar sus interpretaciones parecen ofrecer eficacia y seguridad en su administración.

Tal es la repercusión que podría suponer aclarar esta "pregunta de investigación"; se han escrito varios metaanálisis para intentar contestarla. No obstante, estos metaanálisis se realizan con poblaciones y objetivos distintos, incluyen pacientes con SCA para prevención primaria o secundaria con administración de distintos fármacos en distintas dosis y con intervalos de tiempo en su administración muy distinta. Probablemente, si existieran datos claros y concluyentes apoyados en adecuados ensayos clínicos, sabríamos la utilidad de las estatinas. No obstante, podríamos seguir teniendo dudas, dado que los ensayos clínicos dan información en situaciones utópicas e idóneas. Probablemente la contestación a esta pregunta debería realizarse con un nuevo ensayo clínico dirigido específicamente hacia la administración precoz de estatinas durante la fase aguda del IAM. No obstante, esto supondría un coste de difícil asunción para las empresas farmacéuticas, dado que el objetivo sería la mortalidad en respuesta a una administración precoz (primeras $24 \mathrm{~h}$ ) y ya sabemos que la administración antes del alta hospitalaria es segura, beneficiosa y recomendada por las guías terapéuticas. Los estudios poblacionales realizados sobre registros clínicos tienen el inconveniente de poder tener sesgos, pues no se controlan, pero nos dan una información veraz al reflejar la práctica clínica real y habitual de nuestro medio. Estos registros de cohortes podrían darnos la respuesta a la utilización precoz de las estatinas durante la fase aguda del IAM. Un resultado interesante obtenido del análisis de dichos registros es la elevada administración durante la fase aguda, a pesar de la falta de recomendación; hecho que contrasta con la menor adhesión al tratamiento de otros fármacos recomendados como indicación I, como por ejemplo los bloqueadores beta. La utilización de técnicas de regresión logística nos permite aproximarnos a la causalidad. Además, técnicas matemáticas como el propensity score analysis nos permiten disminuir los sesgos, al homogeneizar los distintos grupos por "intención de tratar", con lo que los resultados obtenidos por estos registros clínicos tienen bastante capacidad de establecer causalidad y aproximarse a los hallazgos obtenidos en los ensayos clínicos. En esta última línea se han publicado registros que tratan de evaluar el efecto de la administración precoz de estatinas en el SCA; no obstante, volvemos a encontrar resultados contradictorios, probablemente debido a la existencia de amplias diferencias metodológicas. La conclusión general de todos los estudios existentes es que la iniciación temprana del tratamiento con estatinas en pacientes con SCA, con o sin la adecuada revascularización, podría aportar un beneficio de mortalidad. Su administración durante la fase aguda del SCA se muestra claramente segura, y muy posiblemente eficiente; por lo tanto, podría plantearse que las distintas sociedades científicas reconsideren la recomendación de la administración precoz de estatinas durante la fase aguda del SCA.

\section{Bibliografía}

1. Endo A. A gift from nature: the birth of the statins. Nat Med. 2008;14:1050-2.

2. Vagelos PR. Are prescription drug prices high? Science. 1991;252:1080-4.

3. LIPID Investigators. Prevention of cardiovascular events and death with pravastatin in patients with coronary heart disease and a broad range of initial cholesterol levels: the Long-Term Intervention with Pravastatin in Ischaemic Disease (LIPID) study group. N Engl J Med. 1998;339:1349-1357.

4. Downs JR, Clearfield M, Weis S, Whitney E, Shapiro DR, Beere $\mathrm{PA}$, et al. Primary prevention of acute coronary events with lovastatin in men and women with average cholesterol levels: results of AFCAPS/TexCAPS: Air Force/Texas Coronary Atherosclerosis Prevention Study. JAMA. 1998;279:1615-22.

5. Maron DJ, Fazio S, Linton MF. Current perspectives on statins. Circulation. 2000;101:207-13.

6. Shepherd J, Cobbe SM, Ford I, Isles CG, Lorimer AR, MacFarlane $\mathrm{PW}$, et al. Prevention of coronary heart disease with pravastatin in men with hypercholesterolemia. N Engl J Med. 1995;333:1301-7.

7. Scandinavian Simvastatin Survival Study Group. Randomised trial of cholesterol lowering in 4444 patients with coronary heart disease: the Scandinavian Simvastatin Survival Study (4S). Lancet. 1994;344:1383-1389.

8. Sacks FM, Pfeffer MA, Moye LA, Rouleau JL, Rutherford JD, Cole TG, et al. The effect of pravastatin on coronary events after myocardial infarction in patients with average cholesterol levels. N Engl J Med. 1996;335:1001-9.

9. The Long-Term Intervention with Pravastatin in Ischaemic Disease (LIPID) Study Group. Prevention of cardiovascular events and death with pravastatin in patients with coronary heart disease and a broad range of initial cholesterol levels. $\mathrm{N}$ Engl J Med. 1998;339:1349-57.

10. Van de Werf F, Bax J, Betriu A, Blomstrom-Lundqvist C, Crea F, Falk V, et al. Guías de práctica clínica de la Sociedad Europea de Cardiología (ESC). Manejo del infarto agudo de miocardio en pacientes con elevación persistente del segmento ST. Rev Esp Cardiol. 2009;62:e1-47.

11. Pollack CV Jr, Antmant EM, Hollander JE; American College of Cardiology; American Heart Association. 2007 focused update to the ACC/AHA guidelines for the management of patients with ST-segment elevation myocardial infarction: implications for emergency department practice. An Emerg Med. 2008;52:344-55.e1. Epub 2008 jun 2.

12. Patel PD, Arora RR. Practical Implications of ACC/AHA 2007 Guidelines for the Management of Unstable Angina/Non-ST Elevation Myocardial Infarction. Am J Ther. 2009 May 19 [Epub ahead of print].

13. Expert panel on detection, evaluation, and treatment of high blood cholesterol in adults. Executive summary of the third report of the National Cholesterol Education Program (NCEP) expert panel on detection, evaluation, and treatment of high blood cholesterol in adults (adult treatment panel III). JAMA. 2001;285:2486-97.

14. Schwartz GG, Olsson AG, Ezekowitz MD, Ganz P, Oliver MF, Waters D, et al. Myocardial Ischemia Reduction with Aggressive Cholesterol Lowering (MIRACL) Study Investigators. Effects of atorvastatin on early recurrent ischemic events in acute coronary syndromes: the MIRACL study: a randomized controlled trial. JAMA. 2001;285:1711-8. 
15. De Lemos JA, Blazing MA, Wiviott SD, Lewis EF, Fox KA, White $H D$, et al. A to $Z$ investigators. Early intensive vs a delayed conservative simvastatin strategy in patients with acute coronary syndromes: phase $\mathrm{Z}$ of the $\mathrm{A}$ to $\mathrm{Z}$ trial. JAMA. 2004;292:1307-16.

16. Van der Harst P, Voors AA, Van Veldhuisen DJ. Intensive frente a moderate lipid lowering with statins after acute coronary syndromes. N Engl J Med. 2004;351:714-7.

17. Liem AH, Van Boven AJ, Veeger NJ, Withagen AJ, Robles de Medina RM, Tijssen JG, et al. Fluvastatin on Risk Diminishment after Acute Myocardial Infarction Study Group. Effect of fluvastatin on ischaemia following acute myocardial infarction: a randomized trial. Eur Heart J. 2002;23:1931-7.

18. Thompson PL, Meredith I, Amerena J, Sloman JG, Harris PJ. Pravastatin in Acute Coronary Treatment (PACT) investigators. Effect of pravastatin compared with placebo initiated within 24 hours of onset of acute myocardial infarction or unstable angina: the Pravastatin in Acute Coronary Treatment (PACT) trial. Am Heart J. 2004;148:e2.

19. Cannon CP, Steinberg BA, Murphy SA, Mega JL, Braunwald E. Meta-analysis of cardiovascular outcomes trials comparing intensive frente a moderate statin therapy. J Am Coll Cardiol. 2006;48:438-45.

20. Hulten E, Jackson JL, Douglas K, George S, Villines TC. The effect of early, intensive statin therapy on acute coronary syndrome: a meta-analysis of randomized controlled trials. Arch Intern Med. 2006;166:1814-21.

21. Briel M, Schwartz GG, Thompson PL, De Lemos JA, Blazing MA, Van Es GA, et al. Effects of early treatment with statins on short-term clinical outcomes in acute coronary syndromes: a meta-analysis of randomized controlled trials. JAMA. 2006;295:2046-56.
22. Stenestrand U, Wallentin L. Swedish Register of Cardiac Intensive Care (RIKS-HIA). Early statin treatment following acute myocardial infarction and 1-year survival. JAMA. 2001;285:430-6.

23. Aronow HD, Topol EJ, Roe MT, Houghtaling PL, Wolski KE, Lincoff $A M$, et al. Effect of lipid-lowering therapy on early mortality after acute coronary syndromes: an observational study. Lancet. 2001;357:1063-8.

24. Newby LK, Kristinsson A, Bhapkar MV, Aylward PE, Dimas AP, Klein WW, et al. Early statin initiation and outcomes in patients with acute coronary syndromes. JAMA. 2002;287:3087-95.

25. Heeschen C, Hamm CW, Laufs U, Snapinn S, Böhm M, White HD. Platelet Receptor Inhibition in Ischemic Syndrome Management (PRISM) Investigators. Withdrawal of statins increases event rates in patients with acute coronary syndromes. Circulation. 2002;105:1446-52.

26. Spencer FA, Allegrone J, Goldberg RJ, Gore JM, Fox KA, Granger $\mathrm{CB}$, et al. GRACE investigators. Association of statin therapy with outcomes of acute coronary syndromes: The GRACE study. Ann Intern Med. 2004;140:857-66.

27. Fonarow GC, Wright RS, Spencer FA, Fredrick PD, Dong W, Every $\mathrm{N}$, et al. Effect of statin use within the first $24 \mathrm{~h}$ of admission for acute myocardial infarction on early morbidity and mortality. Am J Cardiol. 2005;96:611-6.

28. Lenderink T, Boersma E, Gitt AK, Zeymer U, Wallentin L, Vande Werf $\mathrm{F}$, etal. Patients using statin treatment with in $24 \mathrm{~h}$ after admission for ST-elevation acute coronary syndromes had lower mortality than non-users: a report from the first Euro Heart Survey on acute coronary syndromes. EurHeart J. 2006; 27:1799-804. Epub 2006 Jul 4. 